\title{
Phenotypic and functional heterogeneity of peripheral $\gamma \delta$ T cells in pulmonary TB and HIV patients in Addis Ababa, Ethiopia
}

\author{
Mikias Negash ${ }^{1,2^{*}}$, Aster Tsegaye ${ }^{1}$, Liya Wassie ${ }^{2}$ and Rawleigh Howe ${ }^{2}$
}

\begin{abstract}
Background: Previous studies reported HIV infection alters the distribution and function of $\gamma \delta \mathrm{T}$ cells and their subsets. $\delta T$ phenotypes in healthy and diseased individuals has received little attention in Ethiopia. We conducted this study to analyze the distribution of $\gamma \delta T$ cells, the subsets and levels of expression of activation (CD38), exhaustion or anergy (CD95, PD1), adhesion (N-CAM/CD56 and CD103), among HIV and TB infected patients.

Method: The distributions of total $\gamma \delta T$ cells, $V \delta 1$ and $V \delta 2 T$ cells subsets were analyzed in clinical samples collected from asymptomatic HIV, pulmonary TB patients and apparently healthy controls. Multicolor flow cytometry and IFN- $\gamma$ ELISA were used to assess surface markers and functional responses of V $\delta 2 T$ cells to isopentenyl pyrophosphate stimulation, respectively.
\end{abstract}

Result: A total of 52 study participants were enrolled in this study, 22 HIV + TB-, 10 HIV-TB+ and 20 healthy controls. No significant differences were observed in the distribution of total $\gamma \delta T$ cells and in the proportion of $V \delta 1$ subsets in all study groups, though slightly higher proportions were observed in HIV + TB- patients for the latter, of borderline statistical significance $(p=0.07)$. However, the proportion of V $\delta 2 \mathrm{~T}$ cells, as well as the IFN- $\gamma$ response to IPP stimulation, was significantly reduced in HIV + TB- patients compared to healthy controls $(p<0.002)$. Expression of the activation marker CD38 $(p<0.001)$ and adhesion marker CD103 (aEß3) were significantly higher in the V $\delta 1 \mathrm{~T}$ cell subset among both HIV + TB- $(p=0.013)$ and HIV-TB+ $(p=0.006)$ patients compared to healthy controls. Similarly, exhaustion markers, CD95 and PD1, were significantly higher in these two T cell subsets among both HIV + TB- and HIV-TB+ patients $(p<0.01)$. Interestingly, we also observed an increased proportion of effector memory (CD45RA-CD27-) and effector cytotoxic (CD45RA + CD27-) V82 T cell subsets in HIV negative pulmonary TB patients.

Conclusion: In sum, HIV infection was associated with an increase in $V \delta 1$ and a decrease in the function and frequencies of V $82 \mathrm{~T}$ cells. Moreover, increased effector V $\delta 2 \mathrm{~T}$ cells were observed among HIV negative pulmonary TB patients suggesting a potential role of these $T$ cells in the host response to TB.

Keywords: Peripheral $\gamma \delta T$ cells, V $\delta 1$, V $\delta 2$ T cell subsets, Pulmonary TB, HIV

\section{Background}

$\gamma \delta \mathrm{T}$ cells are minor subset, 1-5\%, among circulating $\mathrm{T}$ cells, but are present in abundance within mucosal lymphoid tissue, therein comprising as much as $50 \%$ of $\mathrm{T}$ cells. The two main subsets are V $\delta 1$ and $V \delta 2 \mathrm{~T}$ cells [1]. The $V \delta 1^{+} \gamma \delta \mathrm{T}$ cells are mainly situated at mucosal sites and respond to non-classical major histocompatibility complex

\footnotetext{
*Correspondence: mikiasn2@gmail.com

${ }^{1}$ College of Health Sciences, Department of Medical Laboratory Science,

Addis Ababa University, Addis Ababa, Ethiopia

${ }^{2}$ Armauer Hansen Research Institute, Addis Ababa, Ethiopia
}

molecules such as MICA and/or MICB expressed on stressed cells [2]. The V $\gamma 9 \mathrm{~V} \delta 2 \mathrm{~T}$ cell subset on the other hand are dominant in the peripheral circulation and respond to phospholigands (non-peptide molecules) derived from potentially diverse microbes, including mycobacteria [3]. A role for $\mathrm{V} \gamma 9 \mathrm{~V} \delta 2$ cells in anti-microbial immune defense is suggested by an increase in the activation and proliferation of these T-cells in response to intracellular pathogens, such as Mycobacterium tuberculosis [3, 4].

In vitro, $\mathrm{V} \gamma 9 \mathrm{~V} \delta 2 \mathrm{~T}$ cells have been shown to possess diverse immune activities. $\mathrm{V} \gamma 9 \mathrm{~V} \delta 2 \mathrm{~T}$ cells showed activation

(C) The Author(s). 2018 Open Access This article is distributed under the terms of the Creative Commons Attribution 4.0 International License (http://creativecommons.org/licenses/by/4.0/), which permits unrestricted use, distribution, and 
and expansion upon culture with HIV-infected lymphocytes [5], and this activation has been associated with direct cytotoxic activity mediated by the Fas/Fas-L interactions and by cytotoxic granules. Indirect anti-microbial activities of $\mathrm{V} \gamma 9 \mathrm{~V} \delta 2 \mathrm{~T}$ lymphocytes include roles in Th1 T-cell polarization and dendritic cell (DC) maturation [6].

Prior studies have demonstrated a reduced expression of CD28 and an increased expression of CD38 and HLA- DR on $\gamma \delta$ T cell populations of HIV patients, findings also seen with conventional $\alpha / \beta$ T cells, suggesting the possibility that all circulating $\mathrm{T}$ lymphocyte share similar phenotypic abnormalities in HIV infected patients [7]. Similarly, HIV infected patients were shown to have an increased level of V $\delta 1[8,9]$ and a decreased level of V82 cells [9-11]. Although there has been no clear conclusion, the increased V $\delta 1 \mathrm{~T}$ cell subset may have been due to redistribution from mucosal areas as a result of changes in cytokine levels [6] rather than HIV antigen driven expansion $[8,12]$. Notably, the reduction in the proportion and function of the $V \delta 2$ subset was marked in patients with advanced AIDS stage, and this partially recovered after active antiretroviral therapy (ART) $[10,11]$.

There are conflicting data on the frequency or functional response of total $\gamma \delta$ and V $\delta 2$ T cells in pulmonary TB patients. Some studies have reported an increase in frequency and function of $\gamma \delta$ T cells and V $\delta 2$ subsets in TB patients [13], while others reported a decrease in V82 subsets frequency and function following whole blood stimulation with phosphoantigen [14]; still others reported no differences when compared to healthy controls $[15,16]$. Given the inconsistency of previous reports, as well as lack of information on these $\mathrm{T}$ cells profile in the Ethiopian setting, the aim of this study was to assess the distribution of $\gamma \delta$ T cells and their subsets Moreover, the expression of activation, adhesion and exhaustion markers was measured to further reveal the possible role these $\mathrm{T}$ cells play in such diseases.

\section{Methods}

\section{Study setting and population}

This cross-sectional study, conducted between February 2015 to June 2016, involved a total of 52 participants recruited from a tertiary health facility, the anti-retroviral treatment (ART) follow up clinic of the All African Leprosy and Rehabilitation Training Center (ALERT Hospital), Addis Ababa and two additional medium sized health centers, Arada Health Center and Teklehaimanot Health Center in Addis Ababa.

Twenty two of the participants were HIV positive TB negative patients and 10 were HIV negative pulmonary TB patients. The HIV positive TB negative cases were all asymptomatic, free of any opportunistic infections and were on HAART for at least 2 years before inclusion into the study, whereas the HIV negative TB patients were treatment-naïve pulmonary $\mathrm{TB}$ patients, presenting with clinical TB signs and symptoms and confirmed with acid fast bacilli (AFB) smear microscopy. Additionally, 20 age and sex matched apparently healthy individuals were recruited as controls from Voluntary Counseling Testing centers (VCT). Healthy controls with acute illness, chronic disease conditions such as diabetes, hematological malignancies, HIV and lymphomas were excluded from the study. All participants gave written informed consent and the study received ethical approval from institutional review committee at the Department of Medical Laboratory Science, Addis Ababa University, AHRI/ALERT Ethics Review Committee and the Ethiopian National Research Ethics Review Committee.

\section{Flowcytometry analysis}

Multicolor flowcytometry was used to study the percentage of cells expressing activation, adhesion, exhaustion and memory markers on V $\delta 1$ and $V \delta 2 \mathrm{~T}$ cell subsets in HIV + TB- and HIV-TB+ patients, and healthy controls. Typically, $8 \mathrm{ml}$ of peripheral venous blood was collected from eligible participants in heparin tubes and dispensed and lysed in polystyrene Florescence Activated Cell Sorter (FACS) tubes using red blood cell (RBC) lysis buffer $(0.15 \mathrm{M}$ Ammonium chloride). Following lysis, leukocytes were washed with phosphate buffered saline (PBS), and resuspended in FACS buffer $(1 \mathrm{mg} / \mathrm{ml}$ bovine serum albumin, $1 \mathrm{mM}$ EDTA in PBS). Cells were subsequently stained for cell surface markers using fluorochrome conjugated monoclonal antibodies, namely V $\delta 2-\mathrm{PE}$, TCR $\gamma \delta$-PE, CD56-APC, CD8-APC-Cy7, CD38-V450, CD3-V500, CD95-V450, CD27-V500, CD45RA-PE-Cy7, all from Beckton Dickinson (BD Biosciences, Belgium), V81-FITC (ThermoFisher, South Africa) and PD1-APC, CD103-PE-Cy7 (eBioscience,USA). Cells were incubated for $20 \mathrm{~min}$ at $4{ }^{\circ} \mathrm{C}$, washed with FACS buffer and fixed with $2 \%$ paraformaldehyde. Data was acquired on a FACSCanto II flow cytometer using FACSDiva software (BD Biosciences). Analysis of lymphocyte sub-population frequencies and the relative expression of the different markers was performed with FlowJo Version 9.9.4 Software (TreeStar Inc., USA) after gating on $\mathrm{CD}^{+} \mathrm{T}$

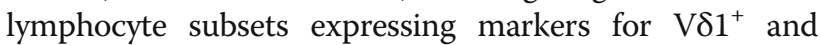
$\mathrm{V} \delta 2^{+}$(see Fig. 2).

IPP-specific IFN- $\gamma$ response whole blood stimulation assay For this experiment, $100 \mu \mathrm{l}$ of heparinized whole blood was dispensed into 96 well plates (BIORAD, USA) in duplicate and was stimulated with or without $50 \mu \mathrm{M}$ isopentenyl pyrophosphate (IPP) (Sigma Aldrich, USA) in RPMI 1640 medium supplemented with 10\% Fetal Calf Serum, 1\% L -glutamine, penicillin, and streptomycin. After $24 \mathrm{~h}$ incubation in $5 \% \mathrm{CO}_{2}$ incubator at $37{ }^{\circ} \mathrm{C}$, 
supernatants were harvested from each well for detection of IPP-specific IFN- $\gamma$ production using an Enzyme Linked Immunosorbent Assay (ELISA) (BIORAD, USA).

The data obtained in the presence of stimuli exhibited Optical Density (OD values above unstimulated controls, but were low and in the non-linear portion of the IFN- $\gamma$ standard curve. Considering the extrapolation of OD values to IFN- $\gamma$ concentrations therefore unreliable, and since all culture supernatants were evaluated by ELISA in the same experiment, we opted to simply express data as OD units in the presence of stimulus less that in the absence of stimulus.

\section{Statistical analysis}

The data was entered into excel, cleaned and imported to Statistical Package for Social Sciences (SPSS) Software Version 21.0 (Chicago, IL, USA) for statistical analyses. Frequencies and proportions were used to describe the characteristics of study participants in relation to relevant variables. The non parametric, Mann-Whitney $U$ test was used to compare median percentage of cell subsets among different study groups. Graphpad Prism Version 6.0 was used to generate graphs. A $p$-value $<0.05$ was considered as statistically significant.

\section{Results}

In this study 52 study subjects participated and were categorized in three groups based on their disease status. The three groups were similar with respect to age and sex. The mean age of the patients was 35.4 and 32.4 for HIV + TB- and HIV-TB+ groups, respectively (Table 1).

\section{Distribution of $\gamma \delta$ T cells and their subsets in peripheral whole blood}

The median percentage of total $\gamma \delta \mathrm{T}$ cells among $\mathrm{CD}^{+}$ $\mathrm{T}$ lymphocytes was comparable in HIV + TB- subjects and healthy controls with respective medians of 3.7\% and $3.5 \%$. HIV-TB+ subjects exhibited a slightly higher median (5.3\%) though this value did not reach statistical significance (Fig. 1). Further delineation into V $\delta 1$ and V $\delta 2$ subsets revealed that while V $\delta 1$ percentages were higher in HIV + TB- than in HC subjects $(p=0.07$, borderline significance), V $\delta 2$ percentages were significantly

Table 1 Socio-demographic characteristics of HIV + TB-, HIV-TB+ patients and healthy controls

\begin{tabular}{|c|c|c|c|c|}
\hline \multirow[t]{2}{*}{ Study Groups } & \multirow[t]{2}{*}{$n$} & \multicolumn{2}{|l|}{ Sex } & \multirow{2}{*}{$\begin{array}{l}\text { Age in years } \\
\text { Mean (SD) }\end{array}$} \\
\hline & & Male & Female & \\
\hline HIV + TB- & 22 & 9 & 13 & $35.4(7.4)$ \\
\hline HIV-TB+ & 10 & 5 & 5 & $32.4(11.5)$ \\
\hline Healthy controls & 20 & 12 & 8 & $31.2(9.4)$ \\
\hline Total & 52 & 26 & 26 & \\
\hline
\end{tabular}

lower. The percentages of total $\gamma \delta \mathrm{T}$ cells among HIV-TB+ subjects did not differ from HC (Fig. 1).

\section{Expression of activation and other markers by $\mathrm{V} \delta 1$ and Vס2 $T$ cells subsets}

We next defined the expression of a range of other markers related to lineage, adhesion, activation, and exhaustion on the two $\gamma \delta$ subsets using a gating strategy as defined in Fig. 2. Our results showed that the median percentage of $\mathrm{V} \delta 1 \mathrm{~T}$ cells expressing CD8 did not significantly differ across all three study groups. However, expression of the activation marker CD38 among V $\delta 1$ cells was significantly higher in both patient groups, HIV + TB- and HIV-TB+, compared to healthy controls $(p<0.001)$. Similarly, the frequency of the adhesion marker CD103 in V $\delta 1$ cells was significantly higher in both patient groups, HIV + TB- $(p=0.013)$ and HIV-TB $+(p=0.005)$, compared to healthy controls. In addition, the expression of exhaustion markers CD95 and PD1 were significantly higher in both patient groups. In contrast, the frequency of CD56 among V $\delta 1$ cells was significantly lower in the patient groups compared to healthy controls (Fig. 3).

Similar to our findings of V $\delta 1 \mathrm{~T}$ cells, the median percentage of CD38 expressing cells within the $\mathrm{V} \delta 2$ subset was significantly higher in the two patient groups than healthy controls $(p<0.001)$, as shown in Fig. 4 . The frequency of cells positive for the exhaustion markers CD95 and PD1 among V $82 \mathrm{~T}$ cells were similarly higher in patients compared to healthy controls $(p=0.001)$. HIV negative TB patients had significantly higher proportions of V $\delta 2 \mathrm{~T}$ cells $(p=0.002)$ and PD1 expressing V82 cells $(p=0.013)$ compared to HIV positive TB negative patients.

\section{Expression of differentiation markers by V $\delta 2 \mathrm{~T}$ cells subsets}

The V $\delta 2 \mathrm{~T}$ cells subsets were further gated and analyzed for the expression of CD27 and CD45RA markers as defined in Fig. 5 to determine the frequency of naïve, memory and effector phenotypes.

The median frequency of central memory (CD45RA-CD27 +) V82 T cells subsets was significantly higher in healthy controls compared to HIV + TB- $(p<0.001)$ and HIV-TB+ $(p=0.02)$ patients. Though not significant, the frequency of naïve (CD45RA + CD27+) V82 T cells was higher in the healthy controls. In contrast, the median frequency of effector cytotoxic (CD45RA + CD27-) V82 $\mathrm{T}$ cells was significantly higher in both patient groups compared to healthy controls $(p=0.001)$. However, we did not find significant differences in the frequency of intermediate effector/effector memory (CD45RA-CD27-) V82 T cells between HIV positive patients and controls. In addition, a higher proportion of 


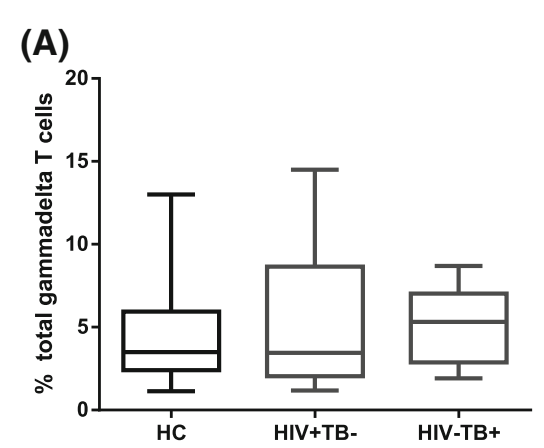

(B)

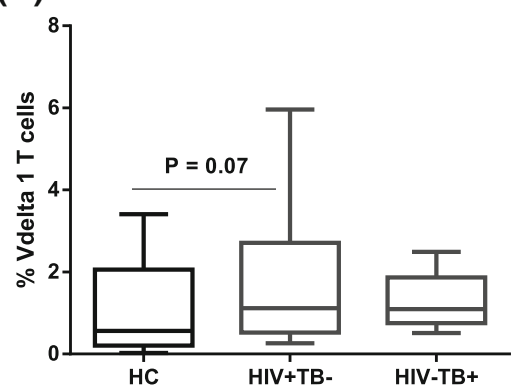

(C)

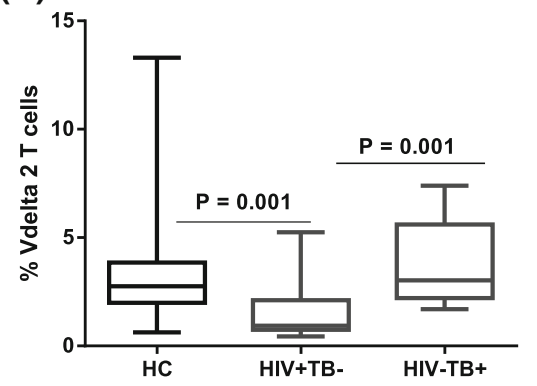

Fig. 1 Distribution of total and subsets of gamma-delta T cells. Distribution of total $\gamma \delta(\mathbf{a}), \mathrm{V} \delta 1$ (b) and V $\delta 2 T$ cells (c). Data shown are box plots depicting median, intra-quartile range and range of subset frequencies defined from peripheral blood cells of 22 HIV + TB- and 10 HIV-TB+ patients, and 20 healthy controls. $p$ values are from the Mann Whitney U Test

these cells was observed in HIV negative pulmonary TB patients $(p=0.034)$ than healthy controls (Fig. 6).

\section{Functional response of $\mathrm{V} \delta 2 \mathrm{~T}$ cells}

The cytokine functional response of $\mathrm{V} \delta 2 \mathrm{~T}$ cells was evaluated by measuring the level of IFN- $\gamma$ production following overnight stimulation with IPP. HIV + TB- patients had the lowest IFN- $\gamma$ OD level compared to healthy controls and HIV-TB+ patients; no significant difference was observed between HIV-TB+ patients and controls (Fig. 7).

\section{Discussion}

The distribution of total $\gamma \delta$ T cells, and V $\delta 1$ and $V \delta 2$ subsets was analyzed in the peripheral blood of HIV and pulmonary TB patients and compared with values from apparently healthy individuals. We also analyzed the expression of activation and adhesion markers on $V \delta 1 \mathrm{~T}$ cells, and exhaustion and differentiation markers and phosphoantigen-specific IFN- $\gamma$ responses of $\mathrm{V} \delta 2$ cells. The median percentage of total $\gamma \delta$ T cells was similar between the patients and healthy controls which is in agreement with previous reports $[8,9]$.

The current study demonstrated an increased proportion of V $\delta 1$ cells in HIV + TB- patients, though of statistically marginally significance compared to controls. Similar results were reported in previous studies, but with greater statistical significance $[17,18]$. On the other hand the frequency of $\mathrm{V} \delta 2$ cells was significantly lower in HIV + TB- patients compared to healthy controls and

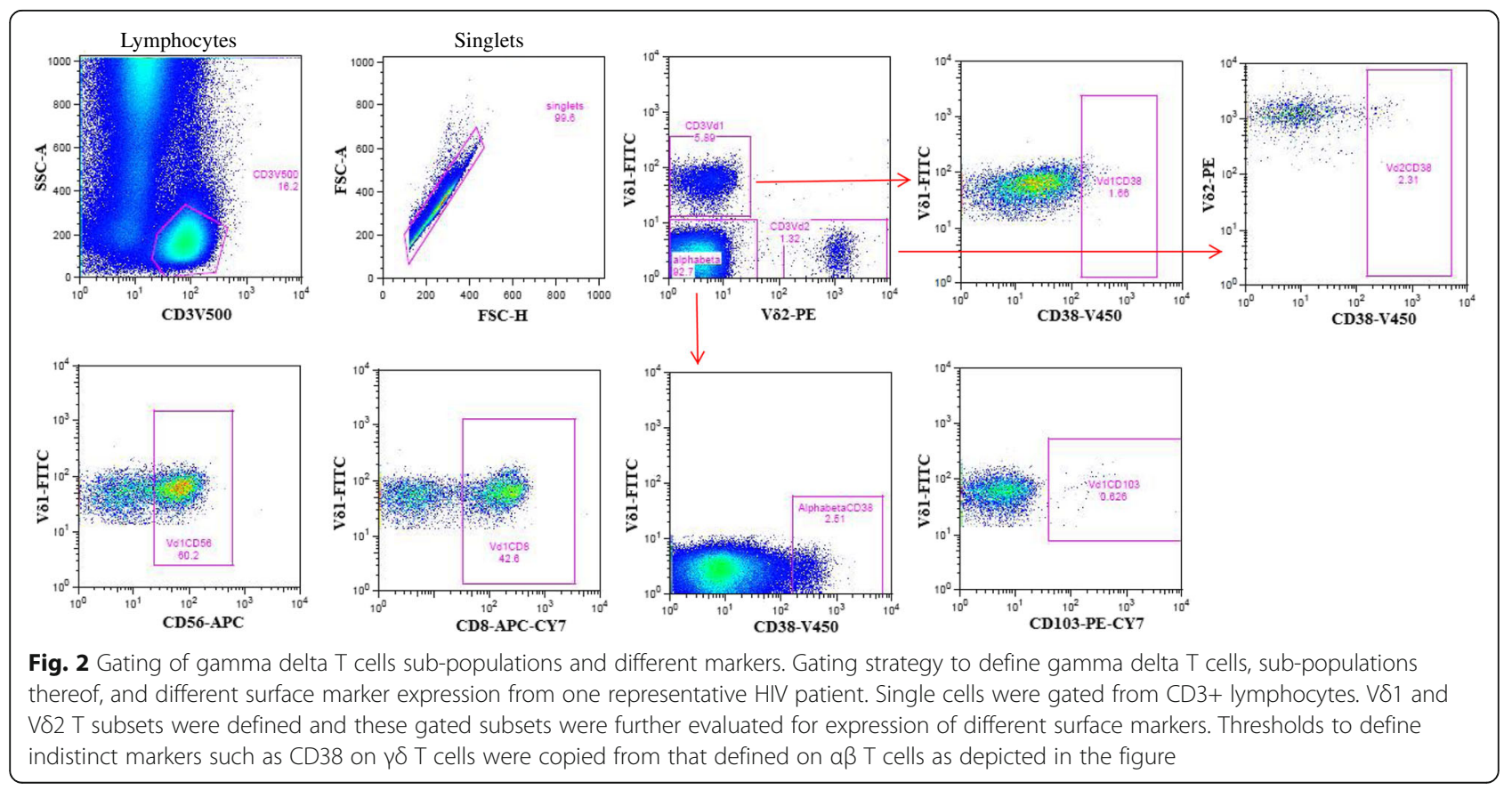


$\mathrm{V}$ delta $1 \mathrm{~T}$ cells

(A)

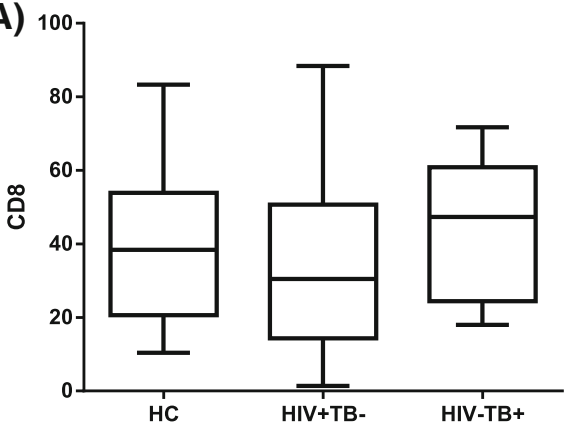

(C)

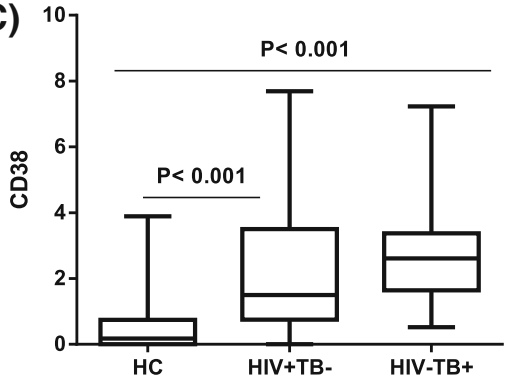

(E)

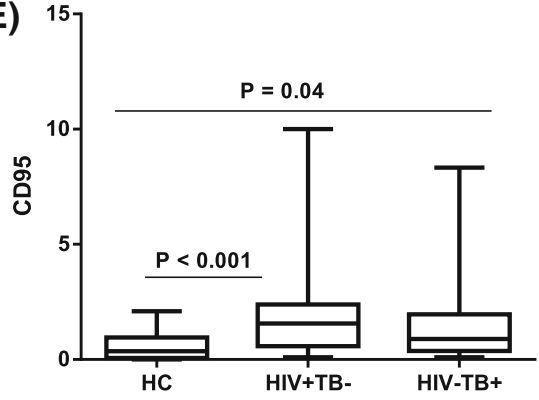

(B)

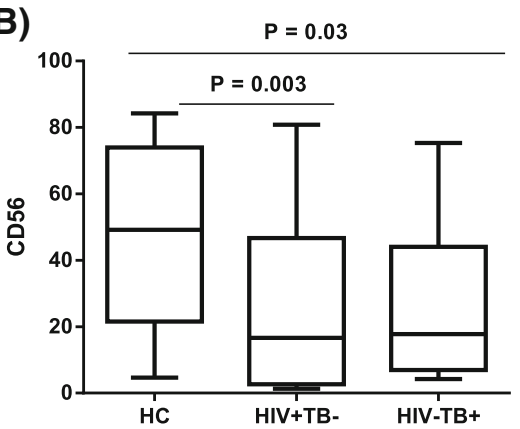

(D)

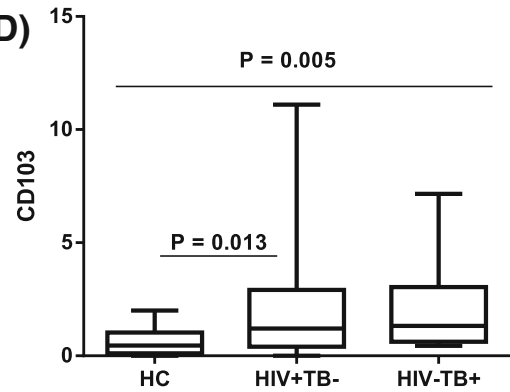

(F)

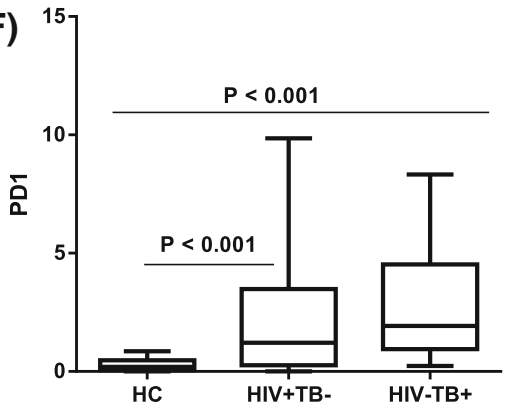

Fig. 3 Expression of different biomarkers on V81 subsets. Median percentage of CD8 (a), CD56 (b), CD38 (c), CD103 (d), CD95 (e), and PD1 (f) on V $81 \mathrm{~T}$ cell subsets from peripheral blood cells of 22 HIV + TB-, 10 HIV-TB+ patients and 20 healthy controls. $p$ values are from the Mann Whitney $\cup$ Test

$\mathrm{V}$ delta $2 \mathrm{~T}$ cells
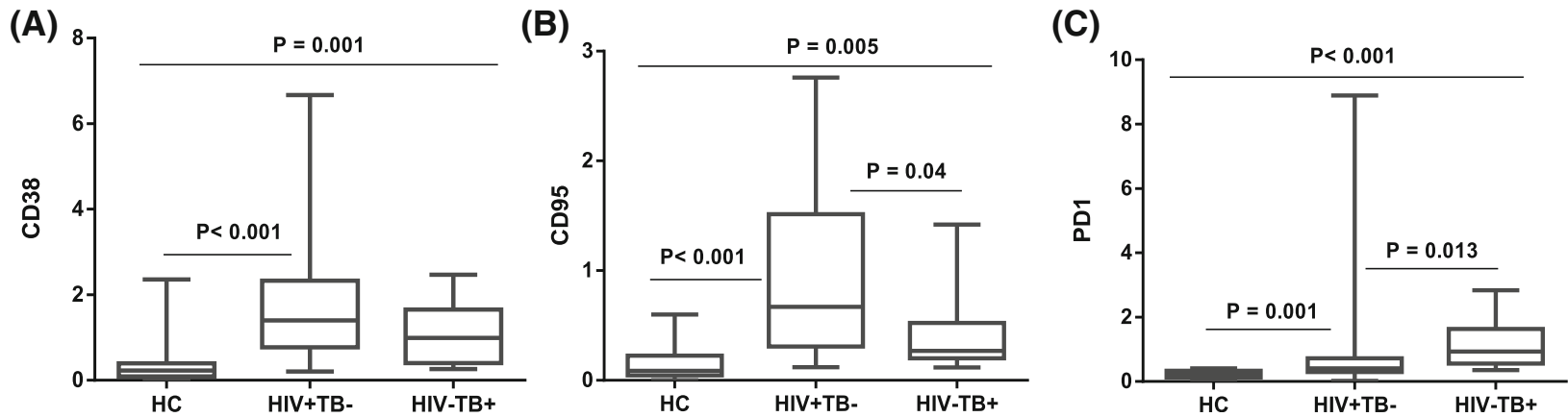

Fig. 4 Expression of different biomarkers on V 82 subsets. Median percentage expression of CD38 (a), CD95 (b) and PD1 (c) on V82 T cell subsets from peripheral blood cells of 22 HIV + TB-, 10 HIV-TB+ patients and 20 healthy controls. $p$ values are from the Mann Whitney U Test 

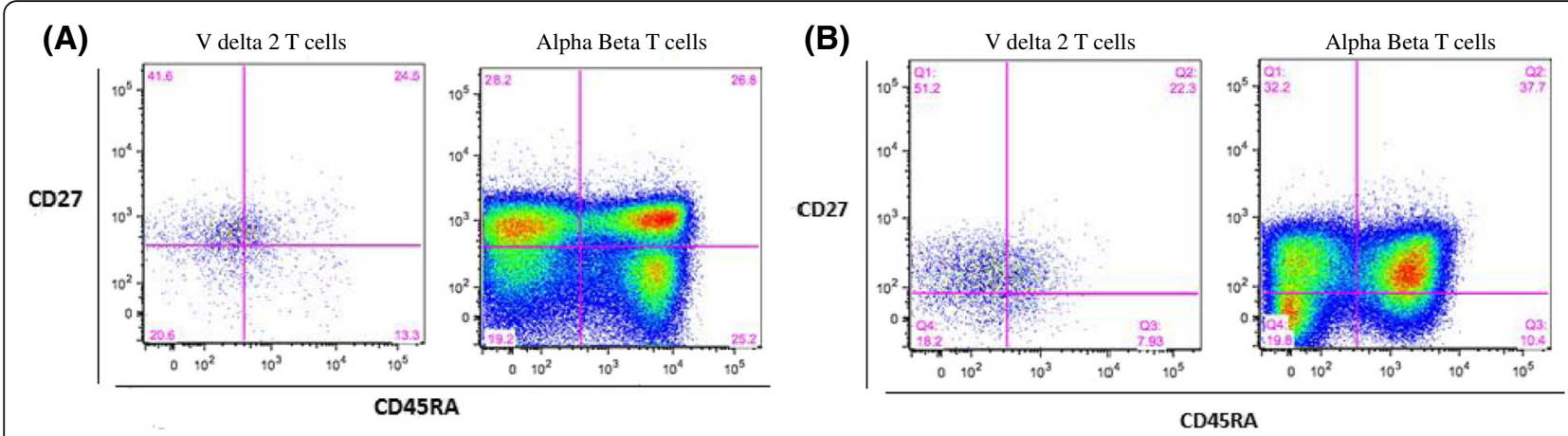

Fig. 5 Gating of memory markers on conventional T cells and gamma delta T cells. Frequency of V82 cells stained with CD27 and CD45RA mAbs. Peripheral blood cells from 22 HIV patients, 10 pulmonary TB patients and 20 controls were stained. Left panel (a) shows a representative pseudocolor plot of CD27 versus CD45RA staining among V82 T cells and a $\mathrm{T}$ cells from one HIV patient. Right panel (b) depicts bivariate staining of CD27 and CD45RA among V82 T cell and a $\mathrm{T}$ cells from one representative healthy control. Notably, as shown in the figure (Fig. 5), thresholds discriminating subsets on the basis of CD27 and CD45RA were defined on a $\mathrm{T}$ cells and copied to plots expressing these markers on gated V82 T cells

HIV-TB+ patients in the current study, such observation was also reported by previous findings $[9,17]$.

There is no clear consensus with regard to the distribution of V $\delta 2$ T cells in HIV negative pulmonary TB patients and healthy controls. Li et al. [19] showed significantly lower V82 T cells number in pulmonary TB patients compared to controls, Carvalho et al. also reported significantly lower values of these $\mathrm{T}$ cells in pulmonary $\mathrm{TB}$ patients irrespective of their HIV status [20]. In contrast, there are reports describing increased proportion of $\mathrm{V} \delta 2 \mathrm{~T}$ cell subsets in pulmonary TB patients co-infected with HIV [21]. Other studies found no difference in these $\mathrm{T}$ cells distribution between pulmonary TB patients and healthy controls [16], similar to our findings in this study. Possibly, the lack of

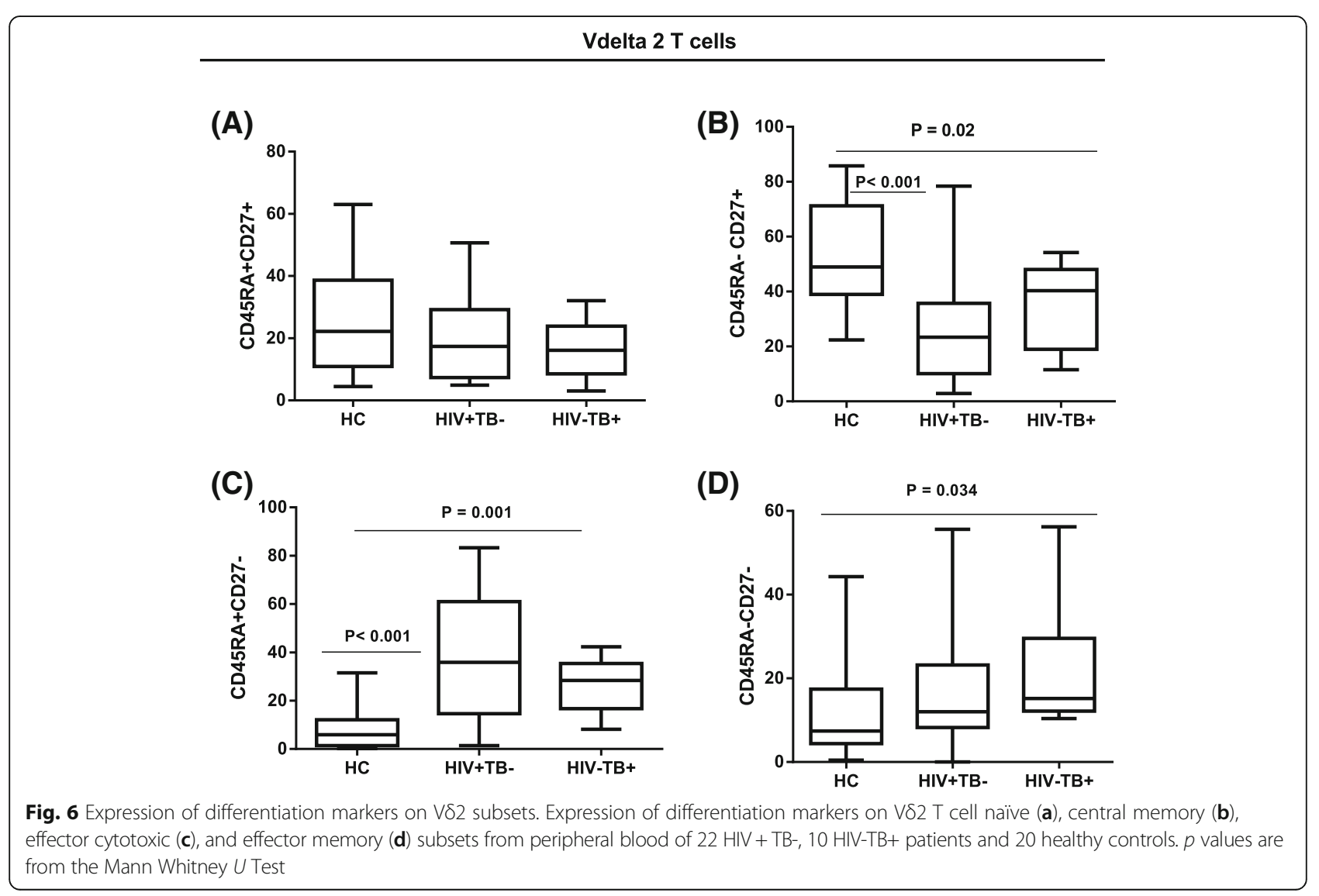




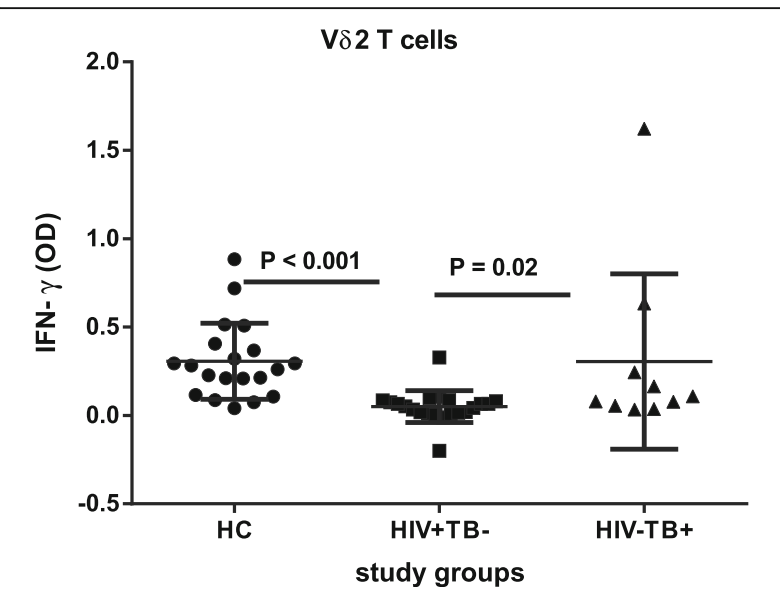

Fig. 7 IFN- $\gamma$ response by V $\delta 2 T$ subsets to Isopentenyl Pyrophosphate stimulation. Comparison of median IFN- $\gamma$ responses by V $82 \mathrm{~T}$ cells after stimulating with $50 \mu \mathrm{M}$ IPP for $24 \mathrm{~h}$ in $22 \mathrm{HIV}+\mathrm{TB}-, 10 \mathrm{HIV}-\mathrm{TB}+$ patients and 20 healthy controls. IFN- $\gamma$ response is depicted as optical density (OD) of the supernatant, as described in materials and methods. $p$-value is from the Mann-Whitney $U$ test

observed consistency in the distribution of V $\delta 2 \mathrm{~T}$ cells in pulmonary TB patients may be related to study dependent differences in the stage of TB disease, or differences in Purified Protein Derivative (PPD) reactivity. Of note, one study showed PPD negative patients had higher counts of $\gamma / \delta \mathrm{T}$ cells than those PPD positive patients or controls [15]. Other studies, however, have shown that the distribution of these cells and their subsets was not associated with response to PPD [20]. Other possibly variables contributing to differences between studies include age; some studies enrolled more of adults $[15,20]$ while other included younger children [16]. The study sample size may contribute as well; we enrolled only limited numbers of pulmonary TB patients, whereas other studies enrolled more subjects $[15,20]$.

Significantly higher proportions of V $\delta 1 \mathrm{~T}$ cells expressed the activation marker CD38 and adhesion marker CD103 in HIV + TB- patients in this study, consistent with previous reports $[7,9]$. This finding is compatible with the involvement of inflammation and stress induced molecules such as MHC class I polypeptide-related sequence A and $\mathrm{B}$ (MICA and MICB) known to stimulate these cells and influence their mucosal attachment [2]. In vitro studies demonstrated that HIV can breach the mucosal barrier and facilitate the translocation of viruses and bacteria [22], potentially contributing to local immune reactivity and promoting emigration of these $\mathrm{T}$ cell subsets into the peripheral blood, possibly through modulation of some chemokine ligands [23]. Supporting this possibility, a recent study revealed an elevated level of a microbial translocation marker sCD14, which was associated with $\gamma \delta$ T cells activation and this activation increased over time [24].
The repertoire analysis of CDR3 length polymorphisms among peripheral and mucosal V $\delta 1 \mathrm{~T}$ cells is an approach which can reveal close lineage relationship of these cells and support the hypothesis of a mucosal activated source of circulating blood V $\delta 1$ cells. Poles et al. study, however, indicated that despite having increased expression of mucosal homing receptors such as CD103 and CCR9 on $\gamma \delta$ $\mathrm{T}$ cells, the repertoires from peripheral blood and mucosal area appeared to represent two distinct populations in HIV patients [9]. Moreover, in our current study the V $\delta 1$ subset also exhibited significant expression of exhaustion markers CD95 and PD1 in HIV patients suggesting persistent activation of these cells and possibly to altered function. Chronic HIV is associated with reduced effector function of mucosal V $\delta 1$ and V $\delta 2$ T-cells [25].

Despite being asymptomatic and on long term HAART, the current research showed that HIV patients have significantly lower percentages of the V $\delta 2$ subset as well as reduced IFN $\gamma$ production in response to phosphoantigen (IPP) stimulation, a finding observed in other studies [26, 27]. One possible mechanism for reduced numbers and function of these cells could be direct infection and destruction by HIV-1 itself, at least among those V 22 expressing CD4 and chemokine receptors CCR5 and CXCR4 [28] or $\alpha 4 \beta 7$ [29]. Of note, increased expression of $\alpha 4 \beta 7$ and CCR 5 at the surface of activated V $\delta 2 \mathrm{~T}$ cells at close proximity has been found to promote the binding of HIV envelope glycoprotein, which ultimately causes significant killing of these subsets [29]. Additional mechanisms are suggested by our observation of significantly higher expression of CD38, CD95 and PD1 on V82 cells which may reflect an on-going exhaustion or initiation of fas-mediated activation induced cell death. These mechanisms are well described to contribute to loss and/or dysfunction of conventional $\alpha / \beta$ CD 4 and CD8 T cells in HIV patients, and have support in studies of $\gamma / \delta$ T cells [30,31].

As demonstrated by previous reports [30] and in the current study, the compartmentalization of V $\delta 2 \mathrm{~T}$ cells to subsets of naïve and memory cells according to the expression of markers such as CD45RA and CD27 is not as clear compared with that of conventional $\alpha / \beta \mathrm{T}$ cells. Using gating defined on $\alpha / \beta$ T cells, we defined V $\delta 2$ T cell subsets as naïve (CD45RA + CD27+), central memory (CD45RA- CD27+), effector memory (CD45RA-CD27-), and effector cytotoxic (CD45RA + CD27-) cells [32]. Previous studies showed HIV patients [30] and pulmonary TB patients [33] had significantly reduced numbers of V82 T cells with the CD45RA-CD27- (effector memory) phenotype. However, in the current and another study [25] there was no significant difference in expression of the effector memory phenotype between HIV patients and healthy controls, and a higher proportion in pulmonary TB patients compared with controls. The overexpression 
of effector memory cells that we observed in TB patients is particularly relevant in light of observations that V $\delta 2$ cells with the effector memory and effector cytotoxic phenotype expressed relatively abundant IFN- $\gamma$ [33] and Granzyme B /perforin [32]. Future studies with more detailed functional characterization of $\mathrm{V} \delta 2$ subsets in TB patients may therefore shed light into their role as an important component of the host response to TB.

\section{Conclusion}

The current study indicated that the frequency of total $\gamma \delta$ T cells was similar among HIV and pulmonary TB patient groups and healthy controls. HIV infection was associated with an increase in the V $\delta 1$ subset and a decrease in the V82 subset frequency and function. Patients with HIV and pulmonary TB infections exhibited significantly higher expression of activation, adhesion and exhaustion markers on V $\delta 1 \mathrm{~T}$ and $\mathrm{V} \delta 2 \mathrm{~T}$ cell subsets. HIV negative pulmonary TB patients had significantly higher proportions of effector V $\delta 2 \mathrm{~T}$ cells, consistent with an active role these $\mathrm{T}$ cells play in mycobacterial defense and/or pathogenesis. This study is the first of its kind in this country which assessed the distribution of these T cells. However, further longitudinal studies evaluating phenotypic and functional properties of $\gamma \delta$ T cells in patients before and after therapy, as well as the inclusion of co-infected patients and larger sample size may help to resolve underlying etiologies contributing to altered distributions of $\gamma \delta \mathrm{T}$ lymphocytes and their role in the pathogenesis of the diseases in these patients.

\begin{abstract}
Abbreviations
AAU: Addis Ababa University; AFB: Acid fast bacilli; AHRI: Armauer Hanson research institute; ALERT: All African leprosy and TB rehabilitation and training center; BD: Becton Dickinson; CCR: Chemokine receptor; CD: Cluster of differentiation; DC: Dendritic cell; ELISA: Enzyme linked immunosorbent assay; FACS : Florescence activated cell sorter; HAART: Highly active antiretroviral therapy; HIV: Human immunodeficiency virus; HLA: Human leukocyte antigen; IFN- $\gamma$ : Interferon gamma; IPP: Isopentenyl pyrophosphate; MHC: Major histocompatibility complex; MTB: Mycobacterium tuberculosis; N-CAM: Neural cell adhesion molecule; OD: Optical density; PBS: Phosphate buffer saline; PD1: Program death; PPD: Purified protein derivative; SPSS: Statistical Package for Social Sciences; TCR: T cell receptor; Th: T helper cell; VCT: Voluntary counseling and testing; $\gamma \delta \mathrm{T}$ : Gamma delta T cell
\end{abstract}

\section{Acknowledgments}

We would like to thank all study participants who volunteered to participate in this study. We also would like to thank the ART clinic and laboratory staff of the All Africa Leprosy and Tuberculosis Rehabilitation and Training Center and the Armauer Hansen Research Institute (AHRI).

\section{Funding}

The study was financially supported by AHRI core fund, received from Norwegian Agency for Development Cooperation (NORAD) and Swedish International Development Cooperation Agency (SIDA). The funders had no role in the study design, conduct, analysis and preparation of the manuscript.

\section{Availability of data and materials}

The dataset generated in the current study are not publicly available because of the nature of the study participant's and its confidentiality, but are available from the corresponding author on reasonable request.

\section{Authors' contributions}

MN, AT, LW and RH conceived, designed the study, analyzed the data and wrote the manuscript. MN conducted the experiment. All the authors read and approved the final version of the manuscript.

\section{Ethics approval and consent to participate}

All participants gave written informed consent and the study received ethical approval from institutional review committee at the Department of Medical Laboratory Science, Addis Ababa University, AHRI/ALERT Ethics Review Committee and the Ethiopian National Research Ethics Review Committee.

\section{Consent for publication}

Not applicable

\section{Competing interests}

The authors declare that they have no competing interests.

\section{Publisher's Note}

Springer Nature remains neutral with regard to jurisdictional claims in published maps and institutional affiliations.

Received: 12 February 2018 Accepted: 24 August 2018

Published online: 15 September 2018

\section{References}

1. Carding SR, Egan PJ. [Gamma][delta] T cells: functional plasticity and heterogeneity. Nat Rev Immunol. 2002;2(5):336-45.

2. Groh V, Steinle A, Bauer S, Spies T. Recognition of stress-induced MHC molecules by intestinal epithelial $\gamma \delta$ T cells. Science. 1998;279(5357):173740.

3. Chen $Z W$, Letvin NL. V $\gamma 2 V \delta 2+T$ cells and anti-microbial immune responses. Microbes Infect. 2003;5(6):491-8.

4. Dieli F, Sireci G, Caccamo N, Di Sano C, Titone L, Romano A, et al. Selective depression of interferon- $y$ and Granulysin production with increase of proliferative response by Vy9/N $22 \mathrm{~T}$ cells in children with tuberculosis. J Infect Dis. 2002;186(12):1835-9.

5. Poccia F, Battistini L, Cipriani B, Mancino G, Martini F, Gougeon ML, et al. Phosphoantigen-reactive Vy9V82 T lymphocytes suppress in vitro human immunodeficiency virus type 1 replication by cell-released antiviral factors including CC chemokines. J Infect Dis. 1999;180(3):858-61.

6. Poccia F, Agrati C, Montesano C, Martini F, Pauza C, Fisch P, et al. Innate Tcell immunity in HIV infections: the role of Vg9Vd2 T lymphocytes. Curr Mol Medic. 2002;2(8):769-81.

7. Le Roy GMFA, JP FM, JP CEHJF, Helluin O, Fukushima N, Bouchart FZC, et al. Similarity of expression of activation markers and CD28 on gamma delta and alpha beta-receptor T cells in HIV infection. Clin Immunol Immunopathol. 1996;79(2):189-93.

8. Hinz T, Wesch D, Friese K, Reckziegel A, Arden B, Kabelitz D. T cell receptor $\gamma \delta$ repertoire in HIV-1-infected individuals. Eur J Immunol. 1994;24(12):3044-9.

9. Poles MA, Barsoum S, Yu W, Yu J, Sun P, Daly J, et al. Human immunodeficiency virus type 1 induces persistent changes in mucosal and blood $\gamma \delta$ T cells despite suppressive therapy. J Virol. 2003;77(19):10456-67.

10. Li H, Peng H, Ma P, Ruan Y, Su B, Ding X, et al. Association between VY2V82 $T$ cells and disease progression after infection with closely related strains of HIV in China. Clin Infect Dis. 2008;46(9):1466-72.

11. Agrati C, D'Offizi G, Gougeon ML, Malkovsky M, Sacchi A, Casetti R, et al Innate gamma/Delta T-cells during HIV infection: Terra relatively incognita in novel vaccination strategies? AIDS Rev. 2011;13:3-12.

12. Boullier S, Cochet M, Poccia F, Gougeon ML. CDR3-independent gamma delta $V$ delta 1+ T cell expansion in the peripheral blood of HIV-infected persons. J Immunol. 1995;154(3):1418

13. Ito M, Kojiro N, Ikeda T, Ito T, Funada J, Kokubu T. Increased proportions of peripheral blood $\gamma \delta T$ cells in patients with pulmonary tuberculosis. Chest 1992;102(1):195-7.

14. Rojas RE, Chervenak KA, Thomas J, Morrow J, Nshuti L, Zalwango S, et al. $V \delta 2+\gamma \delta T$ cell function in Mycobacterium tuberculosis- and HIV-1-positive patients in the United States and Uganda: application of a whole-blood assay. J Infect Dis. 2005;192(10):1806-14.

15. Baliko Z, Szereday L, Szekeres-Bartho J. Gamma/delta T lymphocytes in Mycobacterium tuberculosis infection. Thorax. 1997;52(4):375-7. 
16. Dieli F, Sireci G, Di Sano C, Romano A, Titone L, Di Carlo P, et al. Ligandspecific $a \beta$ and $\gamma \delta$ T cell responses in childhood tuberculosis. J Infect Dis. 2000;181(1):294-301.

17. Wesch D, Hinz T, Kabelitz D. Analysis of the TCR Vgamma repertoire in healthy donors and HIV-1-infected individuals. Int Immunol. 1998;10(8): 1067-75.

18. Rossol R, Dobmeyer JM, Dobmeyer TS, Klein SA, Rossol S, Wesch D, et al. Increase in $V \delta 1+\gamma \delta \mathrm{T}$ cells in the peripheral blood and bone marrow as a selective feature of HIV-1 but not other virus infections. Br J Haematol. 1998; 100(4):728-34

19. Li B, Rossman MD, Imir T, Oner-Eyuboglu AF, Lee CW, Biancaniello R, et al, Disease-specific changes in gammadelta $T$ cell repertoire and function in patients with pulmonary tuberculosis. J Immunol. 1996;157(9):4222-9.

20. Carvalho A, Matteelli A, Airo P, Tedoldi S, Casalini C, Imberti L, et al. $\gamma \delta$ T lymphocytes in the peripheral blood of patients with tuberculosis with and without HIV co-infection. Thorax. 2002;57(4):357.

21. Ruiz P, Geraldino N. Peripheral $\gamma \delta$ T-cell population in HIV-infected individuals with mycobacterial infection. Cytometry. 1995;22(3):211-6.

22. Nazli A, Chan O, Dobson-Belaire WN, Ouellet M, Tremblay MJ, Gray-Owen SD, et al. Exposure to HIV-1 directly impairs mucosal epithelial barrier integrity allowing microbial translocation. PLoS Pathog. 2010;6(4):e1000852.

23. Poggi A, Carosio R, Fenoglio D, Brenci S, Murdaca G, Setti M, et al. Migration of V $\delta 1$ and V $82 T$ cells in response to CXCR3 and CXCR4 ligands in healthy donors and HIV-1-infected patients: competition by HIV-1 tat. Blood. 2004; 103(6):2205-13.

24. Li Z, Li W, Li N, Jiao Y, Chen D, Cui L, et al. $\gamma \delta ~ T$ cells are involved in acute HIV infection and associated with AIDS progression. PLoS One. 2014;9(9): e106064.

25. Cimini E, Agrati C, D'Offizi G, Vlassi C, Casetti R, Sacchi A, et al. Primary and chronic HIV infection differently modulates mucosal V $\delta 1$ and V $\delta 2$ T-cells differentiation profile and effector functions. PLoS One. 2015;10(6):e0129771.

26. Bordon J, Evans PS, Propp N, Davis CE, Redfield RR, Pauza CD. Association between longer duration of HIV-suppressive therapy and partial recovery of the VY2 T cell receptor repertoire. J Infect Dis. 2004;189(8):1482-6.

27. Martini F, Poccia F, Goletti D, Carrara S, Vincenti D, D'Offizi G, et al. Acute human immunodeficiency virus replication causes a rapid and persistent impairment of Vy $9 \mathrm{~V} \delta 2 \mathrm{~T}$ cells in chronically infected patients undergoing structured treatment interruption. J Infect Dis. 2002;186(6):847-50.

28. Imlach S, Leen C, Bell JE, Simmonds P. Phenotypic analysis of peripheral blood $\gamma \delta \mathrm{T}$ lymphocytes and their targeting by human immunodeficiency virus type 1 in vivo. Virology. 2003;305(2):415-27.

29. Li H, Chaudry S, Poonia B, Shao Y, Pauza CD. Depletion and dysfunction of V Y2V82 T cells in HIV disease: mechanisms, impacts and therapeutic implications. Cell Mol Immunol. 2013;10(1):42.

30. Cummings J-S, Cairo C, Armstrong C, Davis CE, Pauza CD. Impacts of HIV infection on Vy2V $22 T$ cell phenotype and function: a mechanism for reduced tumor immunity in AIDS. J Leukoc Biol. 2008;84(2):371-9.

31. Yang Y, Dong B, Mittelstadt PR, Xiao H, Ashwell JD. HIV tat binds Egr proteins and enhances Egr-dependent transactivation of the Fas ligand promoter. J Biol Chem. 2002;277(22):19482-7.

32. Angelini DF, Borsellino G, Poupot M, Diamantini A, Poupot R, Bernardi G, et al. FcyRlll discriminates between 2 subsets of Vy $9 V \delta 2$ effector cells with different responses and activation pathways. Blood. 2004;104(6):1801-7.

33. Gioia C, Agrati C, Casetti R, Cairo C, Borsellino G, Battistini L, et al. Lack of CD27- CD45RA - Vy9V82+ T cell effectors in immunocompromised hosts and during active pulmonary tuberculosis. J Immunol. 2002;168(3):1484-9.

Ready to submit your research? Choose BMC and benefit from:

- fast, convenient online submission

- thorough peer review by experienced researchers in your field

- rapid publication on acceptance

- support for research data, including large and complex data types

- gold Open Access which fosters wider collaboration and increased citations

- maximum visibility for your research: over $100 \mathrm{M}$ website views per year

At $\mathrm{BMC}$, research is always in progress.

Learn more biomedcentral.com/submissions 